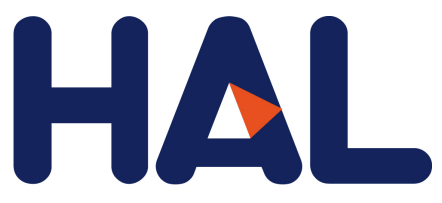

archives-ouvertes

\title{
Continuous theta burst stimulation over the supplementary motor area in refractory obsessive-compulsive disorder treatment: A randomized sham-controlled trial \\ G. Harika-Germaneau, F. Rachid, A. Chatard, C. Lafay-Chebassier, M. \\ Solinas, B. Thirioux, B. Millet, N. Langbour, N. Jaafari
}

\section{To cite this version:}

G. Harika-Germaneau, F. Rachid, A. Chatard, C. Lafay-Chebassier, M. Solinas, et al.. Continuous theta burst stimulation over the supplementary motor area in refractory obsessive-compulsive disorder treatment: A randomized sham-controlled trial. Brain Stimulation, Elsevier, 2019, 10.1016/j.brs.2019.07.019 . inserm-02350724

HAL Id: inserm-02350724

https://www.hal.inserm.fr/inserm-02350724

Submitted on 6 Nov 2019

HAL is a multi-disciplinary open access archive for the deposit and dissemination of scientific research documents, whether they are published or not. The documents may come from teaching and research institutions in France or abroad, or from public or private research centers.
L'archive ouverte pluridisciplinaire HAL, est destinée au dépôt et à la diffusion de documents scientifiques de niveau recherche, publiés ou non, émanant des établissements d'enseignement et de recherche français ou étrangers, des laboratoires publics ou privés. 
Continuous Theta Burst Stimulation over the Supplementary Motor Area in Refractory Obsessive-Compulsive Disorder Treatment: A Randomized Sham-Controlled Trial G. Harika-Germaneau ${ }^{1,2,3}$, F. Rachid ${ }^{4}$, A. Chatard ${ }^{1,2,5}$, C. Lafay-Chebassier ${ }^{2,3,6}$, M. Solinas ${ }^{2,3}$, B. Thirioux ${ }^{1}$, B. Millet ${ }^{7}$, N. Langbour ${ }^{1}$, N. Jaafari ${ }^{1,2,3}$

1. Unité de Recherche Clinique Intersectorielle en Psychiatrie à vocation régionale du Centre Hospitalier Henri Laborit, Poitiers

2. Université de Poitiers

3. INSERM U 1084, Laboratoire de Neurosciences Expérimentales et Cliniques, Poitiers

4. Psychiatrist, Private practice, Place de la Fusterie 7, 1204 Geneva, Switzerland

5. CNRS 7295, Laboratoire CeRCA, Poitiers

6. Service de Pharmacologie clinique et Vigilances, $\mathrm{CHU}$ de Poitiers

7. Département de psychiatrie adulte, ICM-A-IHU, UPMC UMR S 975, Inserm U 1127 , CNRS UMR 7225, GH Pitié-Salpêtrière, Paris, France.

Word-count: 3461

Corresponding author: G. Harika-Germaneau, Pole de Psychiatrie Hospitalier Universitaire Adulte, Unite de recherché Clinique Pierre Denicker, Centre hospitalier henri Laborit, 370 Avenue Jacques Coeur, B.P. 587, 86021 Poitiers Cedex, France, Tel: +33 5165261 18, Fax : + 335494458 31, ghina.harika-germaneau@ch-poitiers.fr 
Introduction

Obsessive-compulsive disorder (OCD) is a complex and clinically heterogeneous neuropsychiatric disorder. It is characterized by recurrent intrusive thoughts causing anxiety and compulsions, that is, repetitive behaviours that aim to relieve distress. The prevalence of OCD is around $2-3 \%$, and OCD symptoms usually emerge during childhood or early adulthood (1). Association of serotonin reuptake inhibitors (SRIs) and cognitive-behavioral therapy (CBT) is the usual treatment of OCD (2). Despite the improvement in pharmacological and behavioral treatments, 40 to $60 \%$ of patient remain resistant to treatment (3). Therefore, developing alternatives to classical therapies may be useful, and neuromodulation techniques offer promising alternatives. Repetitive transcranial magnetic stimulation (rTMS) is a non-invasive technique that has been explored in a number of clinical trials. In recent meta-analyses (4-6), it was found that active rTMS was significantly superior to sham rTMS for the treatment of OCD. Randomized control trials conducted to date focused on three stimulation sites; the left, right or bilateral dorso-lateral prefrontal cortex (DLPFC), the orbito-frontal cortex, and the supplementary motor area (SMA).

In spite of advances in scientific knowledge, the neurobiology of OCD remains poorly understood. The dominant neurobiological model of OCD has implicated dysfunctional cortico-striato-thalamo-cortical (CSTC) circuits in the aetiology of clinical symptoms and cognitive deficits (7). In addition, difficulty in the inhibition process is apparent in the phenomenology of OCD, with patients being apparently unable to stop their obsessions and compulsions. Response inhibition is the ability to inhibit thought and action. It is associated with a main network including the pre-SMA, the inferior frontal gyrus, and the subthalamic nucleus (8). De Wit et al. (9) showed an increased recruitment of the pre-SMA during inhibition task in OCD patients and their healthy siblings, suggesting that hyperactivity in the pre-SMA is a candidate neurocognitive endophenotype of OCD. In 2006, Mantovani et al. (10) showed that inhibition of the SMA has a specific effect in reducing OCD symptoms. Inhibition of the SMA might cause suppression of the hyperexcitable right hemisphere and thereby improves dysfunctional symptoms in patients with OCD. 
According to Rehn et al.'s meta-analysis (6) the SMA is the most relevant cortical target for brain stimulation in OCD patients. To date, five randomized, sham-controlled studies using repeated low-frequency $(1 \mathrm{~Hz})$ stimulation of the SMA in treatment resistant OCD (11-15) have been reported. In these prior studies, low frequency rTMS applied over the SMA showed varying rates of clinical effectiveness. Overall, rTMS yielded a medium effect size in reducing Yale-Brown Obsessive Compulsive Scale (Y-BOCS) scores. Although encouraging, the extant evidence suggests that the treatment of OCD patients with brain stimulation could still be improved.

Over the last ten years, theta burst stimulation (TBS) has been increasingly used as an experimental and therapeutic tool (16). TBS is a form of repetitive transcranial magnetic stimulation (rTMS) involving the use of triple-pulse bursts at $50 \mathrm{HZ}(17)$ repeated five times per second (every 200 milliseconds, $5 \mathrm{~Hz}$ ) in either a continuous (cTBS) or intermittent fashion (iTBS). TBS is thought to induce more rapid and longer-lasting effects on synaptic plasticity than conventional rTMS protocols. It uses lower stimulation intensity and a shorter time of stimulation compared with conventional rTMS protocols (16). cTBS consists of a 40second train of uninterrupted TBS (600 pulses), which, when applied for 40 seconds, decreases cortical excitability for up to 50 minutes, whereas cTBS applied for 20 seconds decreases cortical excitability for 20 minutes $(18,19)$. Application of TBS to the treatment of psychiatric disorders is relatively new, and limited to major depression (20). To date, there is no experimental data or double blind randomized controlled trials on the effectiveness of TBS for reducing clinical symptoms in OCD patients (21). To the best of our knowledge, only one case reports has been published so far (22). This study concerned the efficacy and safety of 10 sessions of cTBS over the right DLPFC in one OCD patient with comorbid depressive disorder.

We report here the results of the first high-powered randomized, double blind shamcontrolled trial testing the effectiveness of cTBS in patients with treatment resistant OCD. Because cTBS induces larger and longer-lasting modulation of cortical excitability than standard rTMS, we expected cTBS protocol over the SMA to reduce OCD symptoms in drug- 
resistant patients. To ensure a high-powered design, we took a number of methodological precautions to provide a stringent test of the hypothesis: we used magnetic resonance imaging to precisely determine in advance the position of the SMA for each patient; all patients received more sessions than in any other trials; and, we included a follow-up period of 6 weeks.

\section{Method}

Study design and randomization

We performed a 6 week randomized, sham-controlled, double blind, parallel group trial that compared the effect of cTBS on OCD symptoms. This trial was conducted in the Poitiers Henri Laborit psychiatric hospital in France. Ethical approval was obtained from the Henri Laborit Ethics Committee. After reading the information sheet and reviewing the potential side effects of rTMS, patients provided their informed consent. Eligible patients were randomly allocated (1:1) to either active cTBS or sham cTBS stimulation using a computed based block randomization (block size of four). The study was conducted between August 2013 and June 2016.

Participants

Thirty outpatients aged between 18 and 65 years, with DSM-IV-TR OCD diagnosed using the Mini-International Neuropsychiatric Interview (MINI) (23) were enrolled in the study. All patients were naïve to TMS. To be eligible, patients had to have a total Y-BOCS score of 20 or more, a total duration of the disease of at least 2 years, and they should have received at least two 12-week adequate sequences and dose of treatment with SRIs but-not responding (treatment resistant). The current medication regimen was maintained throughout the treatment and follow up visits. Benzodiazepines (Lorazepam, Diazepam, or Alprazolam) were also maintained at the same dose throughout the study. Exclusion criteria were as follows: diagnosis of schizophrenia, current major depressive disorder (Montgomery Asberg Depression Rating Scale (MADRS) > 21), other psychotic disorders, bipolar I disorder, substance and alcohol dependence within the last six months, suicidal (score at 3 or more in MADRS, moderate or severe stage in MINI), metallic implant in the cranium (except teeth), 
severe or unstable medical conditions, history of TMS, history of epilepsy, neurological disorders leading to increased intracranial pressure, and severe cardiac disorder and/or with intracardiac lines, cardiac pacemakers and other contraindication to magnetic resonance imaging (MRI). Additionally, participants showing abnormal findings in brain MRI were excluded from the study.

Assessment

Trained psychiatrists who were blind to the patient stimulation group completed clinical assessments. All assessments included the Y-BOCS, the Clinical Global Impression Severity (CGI-S), the MADRS, the Brief Anxiety Scale (BAS), the Global Assessment of Functioning (GAF), the Brown Assessment of Belief Scale (BABS), and the Hospital Anxiety and Depression scale (HAD).

Patients were assessed at baseline, post-cTBS treatment (after 6 week of treatment, W6), and at 6-week follow-up (12 weeks after baseline, W12).

The primary outcome measure was the total Y-BOCS score. Responder status was defined as a $25 \%$ decrease in Y-BOCS score. The secondary outcomes measures were the MADRS, the BABS, the BAS, the CGI-S and the HAD. Before and after each session patients were assessed for TBS side effects with a structured questionnaire.

Treatment

Before the treatment period, each patient had an anatomical T1-weighted magnetic resonance imaging in order to set-up the neuronavigation system (Syneika One; Syneika). The coil was positioned to target the pre-SMA as described in Pelissolo et al. (14). All patients underwent a baseline and weekly evaluation of the motor cortex excitability by measuring the resting motor threshold (RMT). RMT was defined as the minimum magnetic flux needed to elicit a threshold EMG response (50 $\mathrm{mV}$ in peak-to-peak amplitude) in a resting target muscle (Abductor pollicis brevis) in 5/10 trials using single-pulse TMS administered to the contralateral primary motor cortex ${ }^{1}$. 
A total of 30 rTMS sessions were delivered once a day, 5 days a week, for a total of 6 weeks. The cTBS treatment was administered with the MagPro® X100 with Option stimulator (MagVenture, Inc) using the cool B-65 Active/Placebo (A/P) coil which can be configured in active or sham mode by flipping the coil over. Active cTBS was used according to Huang et al. (17) at $70 \%$ of participants' RMT. This intensity was based on previous studies reporting that $70 \%$ of the RMT is equivalent to $80 \%$ of the AMT $(24,25)$. cTBS stimulation consisted of 3 single biphasic pulses separated by $0.02 \mathrm{~s}(50 \mathrm{~Hz})$ repeated every $0.2 \mathrm{~s}(5 \mathrm{~Hz})$ for a total of 600 pulses delivered in a 40 s session.

\section{Sham cTBS}

The sham cTBS was administered using the MagPro ${ }^{\circledR}$ cool B65 A/P coil that is designed to support true double blinded clinical trial. Treatment allocation and parameters were recorded and encrypted in USB-keys using MagPro® MagLink Software v 2.1 for each subject. This software allows the study master to define stimulation parameters (active or sham, pulses configuration, intensity of stimulation) for each participant, to record the stimulation sessions as well as the intensities used during motor threshold and stimulation. With this system the operator cannot modify the rTMS parameters, nor know the patient's randomization arm. In order to maintain the blind condition, we excluded patients who previously received TMS stimulations and also maintained the separation between the rating clinicians and TMS operator'-s.

Dimensional OCD symptoms

OCD symptom dimensions were determined using the statistical algorithm for the extraction of quantitative specific symptom dimension data from the Y-BOCS checklist developed by Schavitt et al.(26). This validated procedure is useful to generate dimensions severity rating from existing Y-BOCS severity scores.

Statistical analyses

Statistical analyses were performed using JASP (https://jasp-stats.org). The demographic and clinical characteristics at baseline were compared between the active cTBS group and sham cTBS group using independent sample t-tests (two-tailed) and chi- 
square tests. Responder status was compared between these two groups using chi-square. Primary and secondary outcomes were analysed using repeated-measures two-way analyses of variance with patient group (active cTBS, sham cTBS) as a between-patient factor and time (baseline, W6, W12), as a within-patient factor. Sphericity was tested using a Mauchly's test. When the assumption was rejected, the Greenhouse-Geisser adjustment was used to adjust the degree of freedom. A significant threshold of $p<0.05$ was chosen for all tests. When statistical significant differences were found in the main effects, post-hoc Bonferroni corrections for multiples comparisons were performed. We also conducted a Bayesian Repeated ANOVA on the Y-BOCS scores to quantify how the observed data supported evidence in favour of the null hypothesis (the absence of difference between active cTBS and sham cTBS).

\section{Results}

Participants

Two patients in the sham cTBS group withdrew after the inclusion visit and before the beginning of the stimulation, leaving 28 patients in the final sample. There were 14 patients in each group (Fig. 1). This sample size provided adequate statistical power $(1-\beta=$ 0.90) to detect the expected within-between interaction in a mixed analysis of variance, based on an observed correlation of 0.60 between repeated measures, and for a medium effect size of $f=0.25$, considered as the minimal clinically important difference (MCID). The statistical power was 0.99 for a large effect size of $f=0.40$.

All patients completed the 30 stimulation sessions and the evaluation visits. Table 1 summarizes the sample characteristics of the study participants at baseline. Baseline demographic, dimensional OCD symptoms, Y-BOCS score and clinical characteristics were similar between groups. A DSM-IV-TR history of major depression was present in $12.5 \%$ of patients in the sham cTBS group and in $14.3 \%$ of patients in the active cTBS group.

At the beginning of the study, pharmacological treatments involved SSRIs $(n=27)$, clomipramine $(n=10)$, other antidepressants $(n=4)$, antipsychotics $(n=16)$, mood stabilisers $(n=5)$, anti-histamine agents $(n=3)$ and benzodiazepines $(n=23)$. Patient's treatments were 
maintained throughout the study and were in adequate and stable dose for more than 12 weeks. Among the patients of both groups, 21 (70 \%) had a current augmentation treatment, with a combination of an antidepressant and either another antidepressant or an antipsychotic or mood stabiliser. Regarding history of drug treatments, $80 \%$ of patients in the active cTBS group and $87 \%$ of patients in the sham cTBS group had received clomipramine in the past or were still receiving it ${ }^{2}$.

\section{Primary outcome}

No differences in responder status between the two groups were found at the posttreatment assessment (active cTBS $=3 / 14,21.4 \%$, sham cTBS $=5 / 14,35.7 \%, p=0.403$ ), at the 6 weeks follow-up visit (active cTBS $=4 / 14,28.4 \%$, sham cTBS $=5 / 14,35.7 \%, p=0.686$ ) and at the 12 weeks follow-up visit (active cTBS $=4 / 14,28.4 \%$, sham cTBS $=5 / 14,35.7 \%$; $p=0.686)$.

The evolution of Y-BOCS scores (Fig. 2) and sub-scores was not significantly different in the two groups between baseline, W6 and W12 (Table 2). For anxiety, depression, and CGI-S there were no significant differences between groups at baseline, W6 and W12 (Table 2).

Repeated measures two-way ANOVA showed a significant main effect of Visit ( $F$ $(2,52)=12.838 ; M S E=14.491 ; p<0.001 ;$ partial $\eta 2=0.331)$, post hoc tests using the Bonferroni correction revealed that mean Y-BOCS scores reduced by an average of $4.214(<0.001)$ at post-treatment visit and then reduced by an average of $4.679(<0.001)$ at follow-up visit. No significant main effect for Group $(F(1,26)=1.045 ; M S E=71.082 ; p=0.316$; partial $\eta 2=0.039)$ and no significant interaction between Group and Visit $(F(2,52)=0.543 ; M S E=14.491$; $p=0.584 ;$ partial $\eta 2=0.02$ ) (Table 2 ) were found. In order to quantify evidence for the null hypothesis, we performed a repeated Bayesian ANOVA (Table 3). A Bayes factor $\left(\mathrm{BF}_{01}\right)$ of 3.909 was found, providing substantial evidence for the null hypothesis that there is no difference in Y-BOCS score between active cTBS and sham cTBS.

Secondary outcomes 
An ANOVA showed no significant interaction between Group and Visit for the $\operatorname{MADRS}(F(2,52)=0.838 ; M S E=23.921 ; p=0.438 ;$ partial $\eta 2=0.031)$, the $B A B S(F(2,50)=0.498$; $M S E=4.264 ; p=0.548 ;$ partial $\eta 2=0.02)$, the BAS $(F(2,52)=0.6 ; M S E=13.403 ; p=0.552 ;$ partial $\eta 2=0.023)$, the CGI-S $(F(2,52)=1.366 ; M S E=0.27 ; p=0.264 ;$ partial $\eta 2=0.05)$ and the HAD $(F(2,52)=0.055 ; M S E=25.284 ; p=0.946 ;$ partial $\eta 2=0.002)($ Table 2$)$.

No severe adverse events were reported during the course of the trial. Mild headache (active cTBS 1 / sham cTBS; 2 ) were the only side effect reported.

\section{Discussion}

The present study is the first randomised trial using cTBS for treating OCD symptoms in treatment resistant patients. Using a relatively large sample of patients and a rigorous and well-controlled protocol, we found no significant difference in Y-BOCS scores between active- and sham cTBS groups. A Bayesian analysis provided stronger evidence for the null hypothesis than for the alternative hypothesis. All in all, our results clearly suggest that cTBS has no discernable efficacy in treating resistant OCD patients. In the present study, cTBS was well tolerated and not associated with side effects, confirming previous findings demonstrating safety and tolerability of cTBS in patients with major depression (20).

In 2006, Mantovani et al. (10) demonstrated that inhibition of the SMA successfully reduced OCD symptoms. Following this pioneering study, 5 randomized trials in OCD using low frequency rTMS over the SMA (11-15) reported inconsistent results. In the current trial, we used cTBS, known to induce larger and longer-lasting modulation of cortical excitability than standard rTMS (16), thus offering the promise of a potentially greater clinical efficacy. Despite this, we found no significant decrease in OCD symptoms.

In a case report, Wu et al. (22) have observed efficacy of theta burst in a 33-year-old OCD patient with depressive comorbidity. In that case report, 10 cTBS sessions (6 sessions twice a week, then 4 sessions once a week) were applied over the right DLPFC. The cTBS parameters were 3 pulses at $50 \mathrm{~Hz}$ with a $200 \mathrm{~ms}$ interval. For the cTBS paradigm, 4 continuous sessions of 20 s TBS trains were given (1,200 pulses) at an intensity of $80 \%$ active motor threshold (AMT). One week later, 10 sessions of iTBS were performed on left 
DLPFC for comorbid depression. These authors report a marked improvement in the symptoms of OCD (Y-BOCS score decreased from 19 to 8), anxiety and depression. This symptomatic improvement was represented by a lack of activation of the right orbitofrontal cortex during a challenge task symptom on MRI after treatment compared to before treatment. Building on this encouraging finding, we tried to use cTBS in order to inhibit the SMA in a relatively large sample of OCD patients. Our results did not confirm our expectations.

We believe there are at least 4 different explanations that may account for our failure to find positive effects of cTBS in the present trial. First, our parameters of stimulation (number of pulses and intensity of the RMT and AMT) may not be optimal. We delivered 600 pulses per session, as recommended by Huang et al. (17) conventional protocol. However, it might not be sufficient to inhibit the SMA. Chistyakov et al. (27) used cTBS over the right DLPFC to treat depressive patients. The result of this open label study suggested that there is a dose effect since the number of stimuli of cTBS enhanced its therapeutic effect.

Chistyakov et al. used 1200 to 3600 pulses per session (600 pulses repeated and separated by a 15 min interval) and found a greater effect for the 3600 pulse (28). In the same way, other studies used a greater number of pulses $(1200$ to 1500$)$ than we did $(11-15,22)$. In our study, 600 pulses may not have been sufficient for inducing clinical effects. However, experimental studies (29) demonstrate that doubling stimulation duration of cTBS may result in a reversal of effect with regard to cortical excitability. Thus, the ideal number of stimulations for best efficacy still needs to be precisely determined.

In addition, we stimulated patients at $70 \%$ of RMT which is approximately equal to $80 \%$ of AMT used in the original protocol of Huang et al. 2005 (17). We chose this intensity in order to guarantee a better tolerability of the treatment (30). However, most popular paradigms used in OCD over SMA are low-frequency tonic stimulation, mostly at $1 \mathrm{~Hz}$, with an intensity of $110-120 \%$ RMT, which is superior to our intensity. Stimulus intensity is very important factor to determine plasticity (31) and highly influences the cTBS after-effects (32). Thus, to date, the best parameters of stimulation of cTBS to obtain maximum efficacy remain 
largely unknown. Further studies are needed to identify the optimal parameters to be used in OCD patients.

Second, our cTBS protocol was designed after previous rTMS protocols as no previous study has examined CTBS in OCD patients. However, it is possible that these two protocols have different effects over the SMA even if they have been shown to exert similar effects on primary motor area (16). Therefore, differences in parameters such as number of pulses, stimulus intensity and time of stimulation need further investigation in order to optimize TBS for clinical applications.

Third, individual differences might contribute to our failure to find a significant effect of cTBS in the present study. Like other methods of non-invasive brain stimulation, the response to TBS protocols is highly variable from one person to another. Genetic factors like BDNF polymorphism (33), difference in intracortical network activation (34), functional connectivity in cortical networks targeted by cTBS stimulation (35) and the effect of patient's age (36) may contribute to explain differential responses to cTBS. In addition, almost all our participants were medicated, and there is some evidence that medication can affect TBSinduced plasticity $(37,38)$. This is another factor that should be considered in future studies. Fourth, all patients included in the present study suffered from severe or very severe OCD. They had high baseline Y-BOCS median scores (30 and 29 in the cTBS and sham groups, respectively), duration of illness (29 and 24 years in the cTBS and sham groups, respectively), and most of the patients were relatively resistant with a mean of 2 SRIs trials and at least 1 augmenting agent before TBS.

The aforementioned factors may have influenced the therapeutic response, and should be taken into account in future studies. Moreover, the blinding procedures could be improved by applying a best guess questionnaire in order to check if the blinding was successfully maintained throughout the trial.

In conclusion, the results of this clinical trial suggest that cTBS over the SMA has no clinically significant effect in treating OCD, even if it was well tolerated. The potential clinical 
utility of cTBS in OCD should be explored in rigorous experimental designs (higher stimulation intensity and number of pulse), and with different inclusion criteria (e.g., less severe and resistant OCD patients, or drug free patients). Availability of novel coil designs ensuring stimulation of much larger and deeper brain region like the double cone-coil (39) have to be considered in future trials. Furthermore, it will be interesting in future studies to use neuroimaging and neurophysiologic techniques in addition to clinical assessments for a better understanding of cTBS neurobiological action. 
Acknowledgment:

The authors thank Maillochaud Sylvie, Lafay Nicolas and Levy Charlotte for their help with this research.

Financial Disclosures:

The authors declare no conflicts of interest related to this study.

\section{References}

1. Ruscio AM, Stein DJ, Chiu WT, Kessler RC. The epidemiology of obsessivecompulsive disorder in the National Comorbidity Survey Replication. Mol Psychiatry. janv 2010;15(1):53-63.

2. Hirschtritt ME, Bloch MH, Mathews CA. Obsessive-Compulsive Disorder: Advances in Diagnosis and Treatment. JAMA. 04 2017;317(13):1358-67.

3. Albert U, Marazziti D, Di Salvo G, Solia F, Rosso G, Maina G. A systematic review of evidence-based treatment strategies for obsessive-compulsive disorder resistant to first-line pharmacotherapy. Curr Med Chem. 22 déc 2017;

4. Trevizol AP, Shiozawa P, Cook IA, Sato IA, Kaku CB, Guimarães FB, et al. Transcranial Magnetic Stimulation for Obsessive-Compulsive Disorder: An Updated Systematic Review and Meta-analysis. J ECT. déc 2016;32(4):262-6.

5. Zhou D-D, Wang W, Wang G-M, Li D-Q, Kuang L. An updated meta-analysis: Short-term therapeutic effects of repeated transcranial magnetic stimulation in treating obsessive-compulsive disorder. J Affect Disord. juin 2017;215:187-96.

6. Rehn S, Eslick GD, Brakoulias V. A Meta-Analysis of the Effectiveness of Different Cortical Targets Used in Repetitive Transcranial Magnetic Stimulation 
(rTMS) for the Treatment of Obsessive-Compulsive Disorder (OCD). Psychiatr Q. 9 févr 2018;

7. Nakao T, Okada K, Kanba S. Neurobiological model of obsessive-compulsive disorder: evidence from recent neuropsychological and neuroimaging findings. Psychiatry Clin Neurosci. août 2014;68(8):587-605.

8. Boehler CN, Appelbaum LG, Krebs RM, Hopf JM, Woldorff MG. Pinning down response inhibition in the brain - Conjunction analyses of the Stop-signal task. Neurolmage. 1 oct 2010;52(4):1621-32.

9. de Wit SJ, de Vries FE, van der Werf YD, Cath DC, Heslenfeld DJ, Veltman EM, et al. Presupplementary motor area hyperactivity during response inhibition: a candidate endophenotype of obsessive-compulsive disorder. Am J Psychiatry. oct 2012;169(10):1100-8.

10. Mantovani A, Lisanby SH, Pieraccini F, Ulivelli M, Castrogiovanni P, Rossi S. Repetitive transcranial magnetic stimulation (rTMS) in the treatment of obsessivecompulsive disorder (OCD) and Tourette's syndrome (TS). Int J Neuropsychopharmacol. févr 2006;9(1):95-100.

11. Gomes PVO, Brasil-Neto JP, Allam N, Rodrigues de Souza E. A randomized, double-blind trial of repetitive transcranial magnetic stimulation in obsessivecompulsive disorder with three-month follow-up. J Neuropsychiatry Clin Neurosci. $2012 ; 24(4): 437-43$.

12. Mantovani A, Simpson HB, Fallon BA, Rossi S, Lisanby SH. Randomized sham-controlled trial of repetitive transcranial magnetic stimulation in treatmentresistant obsessive-compulsive disorder. Int J Neuropsychopharmacol. mars 
2010;13(2):217-27.

13. Hawken ER, Dilkov D, Kaludiev E, Simek S, Zhang F, Milev R. Transcranial Magnetic Stimulation of the Supplementary Motor Area in the Treatment of Obsessive-Compulsive Disorder: A Multi-Site Study. Int J Mol Sci. 22 mars $2016 ; 17(3): 420$.

14. Pelissolo A, Harika-Germaneau G, Rachid F, Gaudeau-Bosma C, Tanguy ML, BenAdhira R, et al. Repetitive Transcranial Magnetic Stimulation to Supplementary Motor Area in Refractory Obsessive-Compulsive Disorder Treatment: a ShamControlled Trial. Int J Neuropsychopharmacol. août 2016;19(8).

15. Arumugham SS, Vs S, Hn M, B V, Ravi M, Sharma E, et al. Augmentation Effect of Low-Frequency Repetitive Transcranial Magnetic Stimulation Over Presupplementary Motor Area in Obsessive-Compulsive Disorder: A Randomized Controlled Trial. J ECT. 12 juin 2018;

16. Suppa A, Huang Y-Z, Funke K, Ridding MC, Cheeran B, Di Lazzaro V, et al. Ten Years of Theta Burst Stimulation in Humans: Established Knowledge, Unknowns and Prospects. Brain Stimulat. juin 2016;9(3):323-35.

17. Huang Y-Z, Edwards MJ, Rounis E, Bhatia KP, Rothwell JC. Theta burst stimulation of the human motor cortex. Neuron. 20 janv 2005;45(2):201-6.

18. Wischnewski M, Schutter DJLG. Efficacy and Time Course of Theta Burst Stimulation in Healthy Humans. Brain Stimulat. août 2015;8(4):685-92.

19. Huang Y-Z, Rothwell JC, Chen R-S, Lu C-S, Chuang W-L. The theoretical model of theta burst form of repetitive transcranial magnetic stimulation. Clin Neurophysiol Off J Int Fed Clin Neurophysiol. mai 2011;122(5):1011-8. 
20. Berlim MT, McGirr A, Rodrigues Dos Santos N, Tremblay S, Martins R.

Efficacy of theta burst stimulation (TBS) for major depression: An exploratory metaanalysis of randomized and sham-controlled trials. J Psychiatr Res. juill 2017;90:102-9.

21. Rachid F. Safety and Efficacy of Theta-Burst Stimulation in the Treatment of Psychiatric Disorders: A Review of the Literature. J Nerv Ment Dis. nov $2017 ; 205(11): 823-39$.

22. Wu C-C, Tsai C-H, Lu M-K, Chen C-M, Shen W-C, Su K-P. Theta-burst repetitive transcranial magnetic stimulation for treatment-resistant obsessivecompulsive disorder with concomitant depression. J Clin Psychiatry. avr 2010;71(4):504-6.

23. Sheehan DV, Lecrubier $\mathrm{Y}$, Sheehan KH, Amorim P, Janavs J, Weiller E, et al. The Mini-International Neuropsychiatric Interview (M.I.N.I.): the development and validation of a structured diagnostic psychiatric interview for DSM-IV and ICD-10. J Clin Psychiatry. 1998;59 Suppl 20:22-33;quiz 34-57.

24. Goldsworthy MR, Pitcher JB, Ridding MC. The application of spaced theta burst protocols induces long-lasting neuroplastic changes in the human motor cortex. Eur J Neurosci. janv 2012;35(1):125-34.

25. Ngomo S, Leonard G, Moffet H, Mercier C. Comparison of transcranial magnetic stimulation measures obtained at rest and under active conditions and their reliability. J Neurosci Methods. 30 mars 2012;205(1):65-71.

26. Shavitt RG, Requena G, Alonso P, Zai G, Costa DLC, de Bragança Pereira CA, et al. Quantifying dimensional severity of obsessive-compulsive disorder for 
neurobiological research. Prog Neuropsychopharmacol Biol Psychiatry. 03 2017;79(Pt B):206-12.

27. Chistyakov AV, Rubicsek O, Kaplan B, Zaaroor M, Klein E. Safety, tolerability and preliminary evidence for antidepressant efficacy of theta-burst transcranial magnetic stimulation in patients with major depression. Int J Neuropsychopharmacol. avr 2010;13(3):387-93.

28. Chistyakov AV, Kreinin B, Marmor S, Kaplan B, Khatib A, Darawsheh N, et al. Preliminary assessment of the therapeutic efficacy of continuous theta-burst magnetic stimulation (cTBS) in major depression: a double-blind sham-controlled study. J Affect Disord. 1 janv 2015;170:225-9.

29. Gamboa OL, Antal A, Moliadze V, Paulus W. Simply longer is not better: reversal of theta burst after-effect with prolonged stimulation. Exp Brain Res. juill $2010 ; 204(2): 181-7$.

30. Oberman L, Edwards D, Eldaief M, Pascual-Leone A. Safety of theta burst transcranial magnetic stimulation: a systematic review of the literature. J Clin Neurophysiol Off Publ Am Electroencephalogr Soc. févr 2011;28(1):67-74.

31. Hamada M, Hanajima R, Terao Y, Arai N, Furubayashi T, Inomata-Terada S, et al. Quadro-pulse stimulation is more effective than paired-pulse stimulation for plasticity induction of the human motor cortex. Clin Neurophysiol Off J Int Fed Clin Neurophysiol. déc 2007;118(12):2672-82.

32. Sasaki T, Kodama S, Togashi N, Shirota Y, Sugiyama Y, Tokushige S-I, et al. The intensity of continuous theta burst stimulation, but not the waveform used to elicit motor evoked potentials, influences its outcome in the human motor cortex. Brain 
Stimulat. avr 2018;11(2):400-10.

33. Chung SW, Hill AT, Rogasch NC, Hoy KE, Fitzgerald PB. Use of theta-burst stimulation in changing excitability of motor cortex: A systematic review and metaanalysis. Neurosci Biobehav Rev. avr 2016;63:43-64.

34. Hamada M, Murase N, Hasan A, Balaratnam M, Rothwell JC. The role of interneuron networks in driving human motor cortical plasticity. Cereb Cortex N Y N 1991. juill 2013;23(7):1593-605.

35. Nettekoven C, Volz LJ, Leimbach M, Pool E-M, Rehme AK, Eickhoff SB, et al. Inter-individual variability in cortical excitability and motor network connectivity following multiple blocks of rTMS. Neurolmage. sept 2015;118:209-18.

36. Freitas C, Perez J, Knobel M, Tormos JM, Oberman L, Eldaief M, et al. Changes in cortical plasticity across the lifespan. Front Aging Neurosci. 2011;3:5.

37. Eggers C, Günther M, Rothwell J, Timmermann L, Ruge D. Theta burst stimulation over the supplementary motor area in Parkinson's disease. J Neurol. févr $2015 ; 262(2): 357-64$.

38. Stagg CJ, Wylezinska M, Matthews PM, Johansen-Berg H, Jezzard P, Rothwell JC, et al. Neurochemical effects of theta burst stimulation as assessed by magnetic resonance spectroscopy. J Neurophysiol. juin 2009;101(6):2872-7.

39. Lu M, Ueno S. Comparison of the induced fields using different coil configurations during deep transcranial magnetic stimulation. PLoS ONE [Internet]. 6 juin 2017 [cité 4 avr 2019];12(6). Disponible sur:

https://www.ncbi.nlm.nih.gov/pmc/articles/PMC5460812/ 
40. Jaafari N, Baup N, Bourdel M-C, Olié J-P, Rotge J-Y, Wassouf I, et al. Neurological soft signs in OCD patients with early age at onset, versus patients with schizophrenia and healthy subjects. J Neuropsychiatry Clin Neurosci. $2011 ; 23(4): 409-16$. 
Figure legends

Fig. 1: The CONSORT flowchart with the number of participants in each phase.

Fig. 2: Evolution of Y-BOCS scores between active and sham cTBS. 
Table 1 Baseline demographic and clinical characteristics of the participants

\begin{tabular}{cccc} 
Variable & Active cTBS $(\mathrm{n}=14)$ & Sham cTBS $(\mathrm{n}=14)$ & p-value \\
\hline Gender (M/F) & $5 / 9$ & $8 / 6$ & $0.256^{\#}$ \\
Age (years) & $46.3(10.1)$ & $48.2(12.9)$ & $0.664^{*}$ \\
Age at onset (years) & $18.1(10.1)$ & $26.1(15.5)$ & $0.129^{*}$ \\
Duration of illness (years) & $29.5(9.4)$ & $23.9(16.3)$ & $0.281^{*}$ \\
Y-BOcs score & & & \\
Total & $30.1(4.4)$ & $29.4(4.7)$ & $0.681^{*}$ \\
Obsession & $14.6(2.5)$ & $14.4(2.9)$ & $0.838^{*}$ \\
Compulsion & $15.5(2.1)$ & $15.0(2.5)$ & $0.564^{*}$
\end{tabular}

Dimensional OCD symptoms

$\begin{array}{cccc}\text { Aggression } & 7.4(5.5) & 8.6(4.3) & 0.520^{*} \\ \text { Sexual / religious } & 1.2(3.1) & 3.2(4.2) & 0.165^{*} \\ \text { Symmetry ordering } & 9.1(5.5) & 10.2(3.4) & 0.541^{*} \\ \text { Cleaning contamination } & 8.8(5.2) & 8.1(5.4) & 0.723^{*} \\ \text { Hoarding } & 3.8(5.0) & 2.1(3.5) & 0.324^{*} \\ \text { CGI-S score } & & & \\ \text { MADRS score } & 5.4(0.5) & 5.5(0.8) & 0.773^{*} \\ \text { BAS score } & 13.8(8.6) & 12.1(5.9) & 0.563^{*} \\ \text { GAF score } & 11.7(8.4) & 11.9(7.9) & 0.945^{*} \\ \text { BABS score } & 48.6(8.4) & 44.2(6.7) & 0.140^{*} \\ \text { HAD score } & 4.6(4.0) & 3.7(2.5) & 0.506^{*} \\ \end{array}$

Notes: Data are presented as mean (SD). Y-BOCS: Yale Brown Obsessive Compulsive Disorder

Scale ; CGI-S: Clinical Global Impressions Severity; MADRS: Montgomery-Asberg Depression Rating Scale; BAS: Brief Anxiety Scale; GAF: Global Assessment of Functioning; BABS: Brown Assessment of Beliefs Scale; HAD: Hospital Anxiety and Depression scale.

* The p-value was obtained by a two sample two tailed t-test

\# The $p$-value was obtained using a Pearson $x^{2}$ two-tailed test 
Table 2: Outcome comparison between active cTBS group and sham cTBS group

\begin{tabular}{|c|c|c|c|c|c|c|c|}
\hline \multirow[b]{2}{*}{ Scales } & \multicolumn{3}{|c|}{ Active cTBS $(n=14)$} & \multicolumn{3}{|c|}{ Sham cTBS $(n=14)$} & \multirow[b]{2}{*}{ p-value (group*visit) } \\
\hline & Baseline & W6 & W12 & Baseline & W6 & W12 & \\
\hline YBOCS & $\begin{array}{l}30.07 \\
(4.38)\end{array}$ & $\begin{array}{l}26.57 \\
(4.35)\end{array}$ & $\begin{array}{l}26.43 \\
(5.80)\end{array}$ & $\begin{array}{l}29.36 \\
(4.70)\end{array}$ & $\begin{array}{l}24.43 \\
(7.44)\end{array}$ & $\begin{array}{l}23.64 \\
(7.14)\end{array}$ & 0.584 \\
\hline CGI & $\begin{array}{c}5.43 \\
(0.51)\end{array}$ & $\begin{array}{c}5.21 \\
(0.43)\end{array}$ & $\begin{array}{c}5.00 \\
(0.56)\end{array}$ & $\begin{array}{c}5.50 \\
(0.76)\end{array}$ & $\begin{array}{c}5.21 \\
(0.89)\end{array}$ & $\begin{array}{c}4.64 \\
(1.01)\end{array}$ & 0.264 \\
\hline MADRS & $\begin{array}{l}13.79 \\
(8.62)\end{array}$ & $\begin{array}{c}8.21 \\
(5.40)\end{array}$ & $\begin{array}{c}9.57 \\
(7.72)\end{array}$ & $\begin{array}{l}12.14 \\
(5.97)\end{array}$ & $\begin{array}{c}9.43 \\
(7.62)\end{array}$ & $\begin{array}{r}10.93 \\
(7.51)\end{array}$ & 0.438 \\
\hline BAS & $\begin{array}{l}11.71 \\
(8.44)\end{array}$ & $\begin{array}{c}7.29 \\
(5.78)\end{array}$ & $\begin{array}{c}7.79 \\
(6.90)\end{array}$ & $\begin{array}{l}11.93 \\
(7.89)\end{array}$ & $\begin{array}{c}8.64 \\
(7.33)\end{array}$ & $\begin{array}{c}7.00 \\
(5.07)\end{array}$ & 0.552 \\
\hline BABS & $\begin{array}{c}4.57 \\
(4.01)\end{array}$ & $\begin{array}{c}4.36 \\
(4.05)\end{array}$ & $\begin{array}{c}3.64 \\
(2.74)\end{array}$ & $\begin{array}{c}3.69 \\
(2.66)\end{array}$ & $\begin{array}{c}4.08 \\
(2.50)\end{array}$ & $\begin{array}{c}3.69 \\
(2.69)\end{array}$ & 0.548 \\
\hline HAD & $\begin{array}{l}21.71 \\
(9.22)\end{array}$ & $\begin{array}{l}17.07 \\
(7.62)\end{array}$ & $\begin{array}{r}17.64 \\
(8.22)\end{array}$ & $\begin{array}{l}21.50 \\
(6.10)\end{array}$ & $\begin{array}{r}17.50 \\
(6.87)\end{array}$ & $\begin{array}{r}18.29 \\
(6.74)\end{array}$ & 0.946 \\
\hline
\end{tabular}

Notes: Data are presented as mean (SD). YBOCS: Yale Brown Obsessive Compulsive Disorder Scale; CGI: Clinical Global Impressions; MADRS: Montgomery-Asberg depression rating scale; BAS: Brief Anxiety Scale; BABS: Brown Assessment of Beliefs Scale; HAD: Hospital Anxiety and Depression scale. 
Table 3: Bayesian repeated-measures ANOVA results.

\begin{tabular}{llllll}
\hline Models & $\mathrm{P}(\mathrm{M})$ & $\mathrm{P}(\mathrm{M} \mid$ data) & $\mathrm{BF}_{\mathrm{M}}$ & $\mathrm{BF}_{01}$ & Error \% \\
\hline Null model (incl. Visit, & 0.5 & 0.796 & 3.909 & 1.0 & \\
Group, subject) & & & & & \\
Visit* Group & 0.5 & 0.204 & 0.256 & 3.909 & 2.327 \\
\hline
\end{tabular}

Notes: $\mathrm{P}(\mathrm{M})$ : indicates prior model probabilities; $\mathrm{P}(\mathrm{M} \mid \mathrm{data})$ : indicates the updated probabilities after having observed the data; $\mathrm{BF}_{\mathrm{M}}$ : indicates the degree to which the data have changed the prior model odds; $\mathrm{BF}_{01}$ : Bayes factor in favor of the null hypothesis. 


\section{Footnotes}

1 RMT was measured independently of subjects' handedness on the same hemisphere. Handedness was determined with the item 20 of the neurological soft signs Kreb's scale (40).

2 Only one patient was left-handed. The results were the same when we excluded this patient from the analyses. 


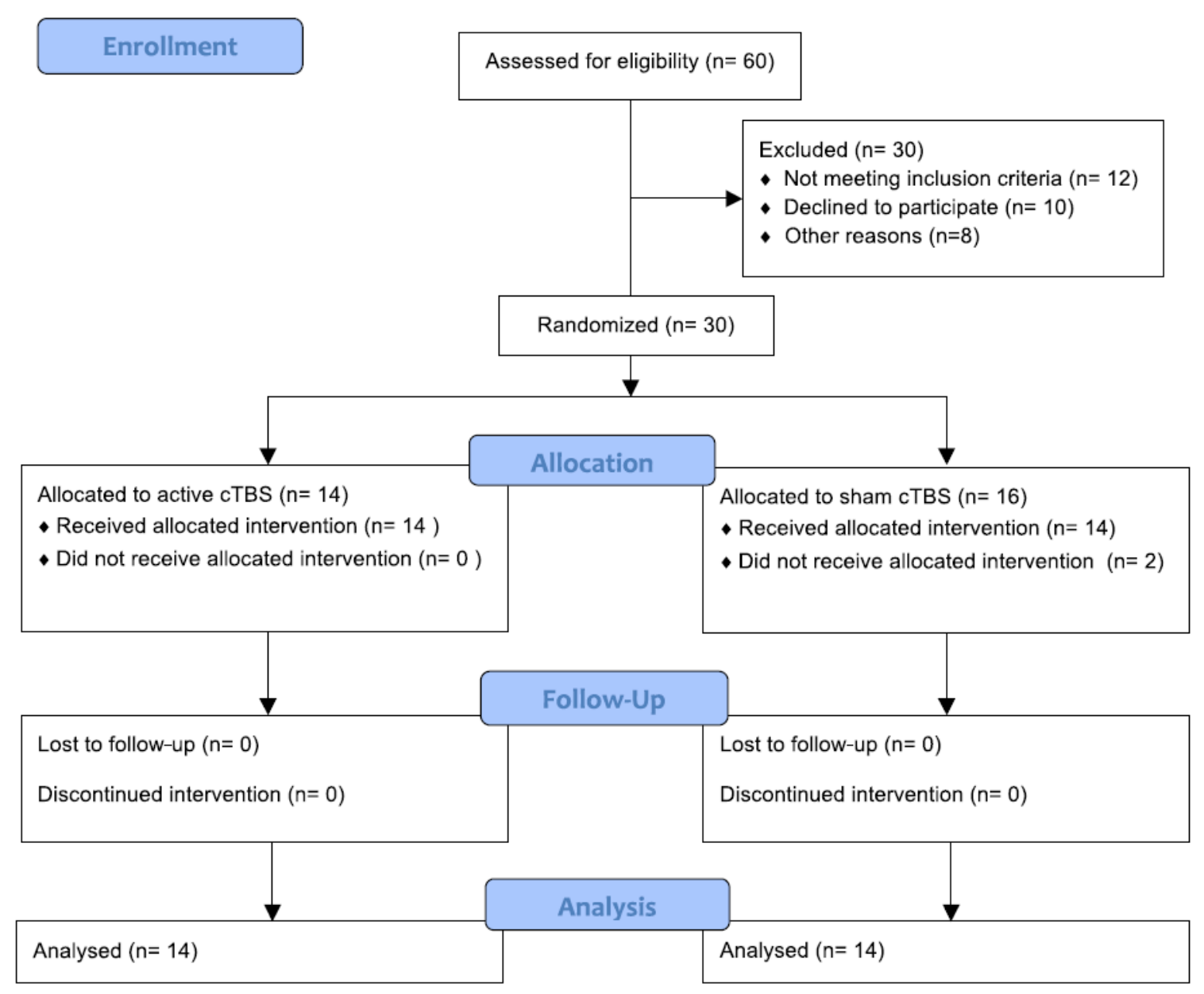

Fig. 1. The CONSORT flowchart with the number of participants in each phase. 


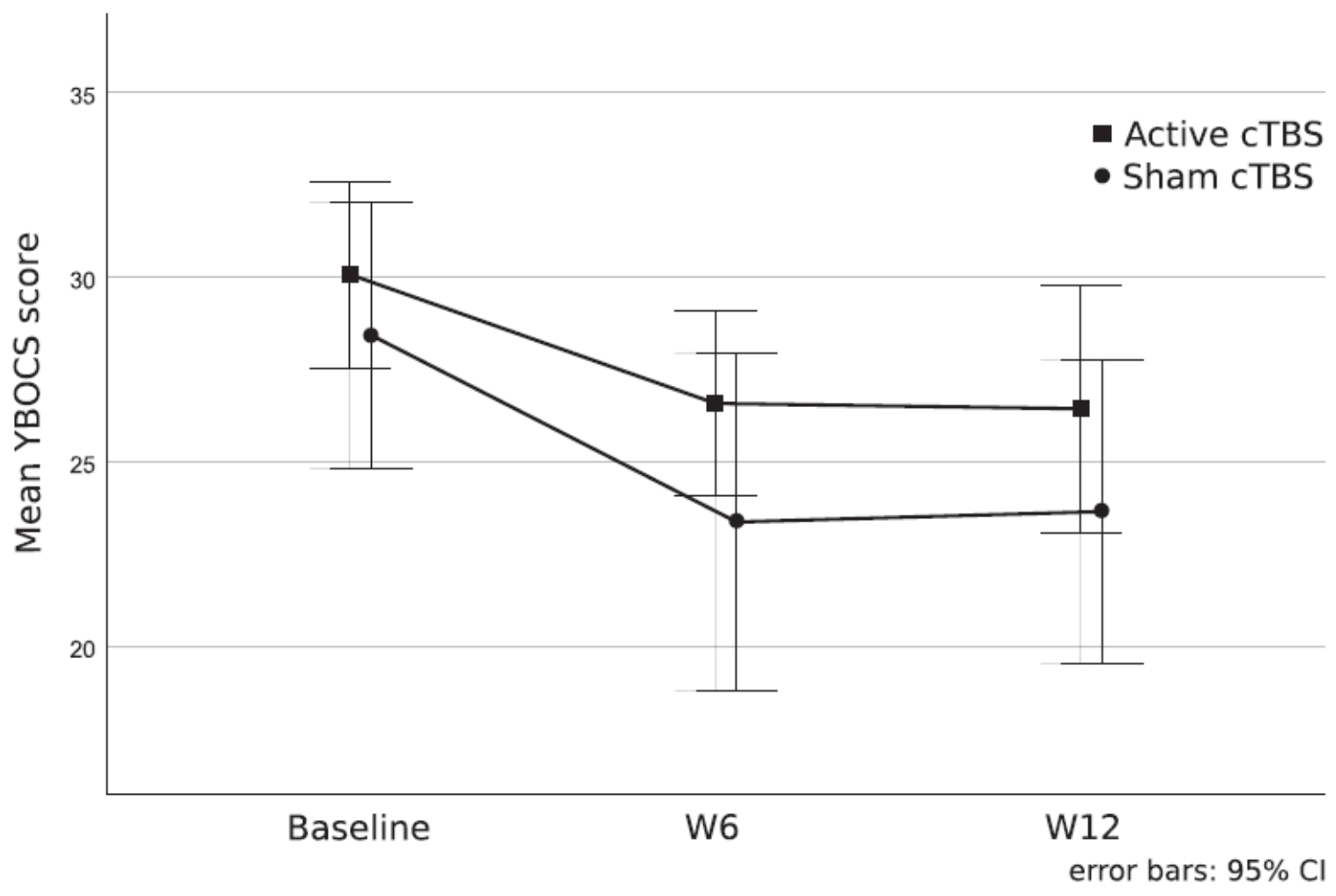

Fig. 2. Evolution of Y-BOCS scores between active and sham cTBS. 